\title{
Polvo de lavar o Irex: incidencia de estímulos fonológicos y semánticos en la producción de palabras habladas de una paciente afásica ${ }^{1}$
}

\author{
Laura Casasa Núñez² \\ Instituto Tecnológico de Costa Rica
}

\section{RESUMEN}

Se estudia la incidencia de los estímulos fonológicos y semánticos en los procesos de producción léxica, a partir de los datos obtenidos en pruebas de denominación con paciente afásica con características anómicas. Arroja datos en relación con la naturaleza del lexicón, el debate entre procesos seriales y de acceso directo y su papel en la recuperación léxica la longitud fonológica y silábica de la palabra.

\begin{abstract}
The incidence of semantic and phonological stimuli in word production processes is addressed. This research analyzes the results obtained from different denomination tasks with an anomic speaker with aphasia. The basis of research was a lexicon theory, the debate between connectionist or serial levels in language production, and the incidence of syllabical and phonological length in word recovery.
\end{abstract}

Palabras clave: Psicolingüística, Neuropsicología, cognición, producción de lenguaje, afasia.

Keywords: Psycholinguistics, Neuropsychology, cognition, language production, aphasia, lexical frequency.

${ }^{1}$ Recibido: 15 de abril 2007; aceptado: 30 de mayo 2009.

${ }^{2}$ Correo electrónico: laura.casasa@gmail.com 


\section{Introducción}

En psicolingüística, se postula la existencia de un sistema de procesamiento de lenguaje, encargado de realizar las tareas de percepción y de producción propias de la conducta humana. Estas tareas se realizan en el plano oral (escuchar y hablar), en el escrito (leer y escribir) y en el motor-visual (lengua de señas) ${ }^{3}$. En los modelos de procesamiento lingüístico se habla frecuentemente de almacenes, que consisten en depósitos de distintas formas lingüísticas, correspondientes a determinados módulos de información. En relación con el léxico, algunos postulan la existencia de cuatro almacenes especializados, mientras que otros defienden la existencia de un único almacén fonológico de entrada y salida ${ }^{4}$.

Esta investigación es el resultado de una serie de entrevistas realizadas con una paciente costarricense (en adelante C.C.) a quien se le diagnosticó una afasia no fluida y cuya capacidad de denominación había sido significativamente disminuida a causa de su enfermedad. La investigación busca aportar datos en relación con el debate teórico sobre la naturaleza del lexicón, a partir del análisis de la incidencia de los estímulos semánticos y fonológicos en la activación de las palabras en procesos de producción. Para este objetivo, se partió de la hipótesis de que si los procesos de producción del habla están organizados por las relaciones semánticas, entonces en pruebas de producción guiadas con estímulos semánticos y fonológicos, la paciente obtendrá un mejor desempeño en aquellas realizadas bajo estímulos semánticos.

El trabajo con pacientes afásicos es una valiosa fuente para el conocimiento del procesamiento lingüístico, pues permite inferir los

$\overline{3}$ Lilianne Manning, Introducción a la neuropsicología clásica y cognitiva del lenguaje. Teoría, evaluación y rehabilitación de la afasia (Madrid: Trotta, 1992); Fernando Cuetos Vega, Evaluación y rehabilitación de las afasias. Aproximación cognitiva (Madrid: Médica Panamericana, 1998).

${ }^{4}$ Cuetos; Alan Allport, «Speech Production and Comprehension: One Lexicon or Two?», Cognition and motor processes (Berlin: Springer-Verlag, 1984). 
procesos subyacentes al fenómeno del habla ${ }^{5}$. El principio de modularidad explica que la información relativa a distintas representaciones lingüísticas está relacionada con segmentos distintos del sistema cognitivo, de manera que si un área sufre alteraciones o daños, las demás continúan desempeñándose eficazmente. En relación con estos aspectos, los datos obtenidos en la entrevista con C.C., nos dan indicios acerca de la composición semántica y fonológica de esta arquitectura del sistema lingüístico.

A partir de estos datos, se ofrece un análisis cualitativo de las emisiones lingüísticas de la paciente, basado en los ejes semántico y fonológico. Se establecen las implicaciones sobre los planteamientos teóricos del sistema cognitivo lingüístico y las aportaciones que arrojan los datos al conocimiento y la descripción del acceso léxico en la producción del lenguaje. La investigación proporciona datos al campo de la afasiología, específicamente, del español de Costa Rica y contrasta los datos obtenidos con las observaciones hechas en otros países, hispanohablantes o no, en relación con los modelos de producción del léxico. Asimismo, aporta evidencias en torno al problema teórico planteado.

\section{Estado de los conocimientos}

En Costa Rica, las principales investigaciones referidas al tema de los trastornos del lenguaje se han desarrollado en las Escuelas de Educación y Psicología, en la Universidad de Costa Rica, y con fines pedagógicos. Algunos textos publicados ofrecen también información al respecto. Hernández $\operatorname{Vargas}^{6}$ da a conocer los resultados de su investigación médica en relación con los traumas cerebrales y el lenguaje. Presenta un informe sobre cuatro pacientes con tumores

\footnotetext{
${ }_{5}^{5}$ Fernando Cuetos, Anomia. La dificultad para recordar las palabras (Madrid: Tea Ediciones, 2003); Jean Berko y Nan Bernstein (Eds.), Psicolingüística (Madrid: McGraw Hill/Interamericana de España, 1999).

${ }^{6}$ Enrique Hernández Vargas, «Afasia como síntoma principal de tumor cerebral maligno», Neuroeje VI (1988): 48-52.
} 
malignos cuyo primer y más importante síntoma fue un trastorno del lenguaje. Para Hernández Vargas, la afasia puede ocurrir en asociación con cualquier tipo de lesión que produzca cambios estructurales en la corteza cerebral o en los tractos asociativos corticales. En este estudio, el autor concluye que una afasia puede ser la primera manifestación de un tumor cerebral maligno, por lo que deben realizarse exámenes exhaustivos al paciente.

El estudio lingüístico de Laganaro y Arrieta $^{7}$ analiza los procesos de activación de la representación fonológica en la producción oral de palabras, desde una perspectiva psicolinguiística, mediante la observación de una paciente afásica hispanófona que produce parafasias fonológicas. Como punto de partida, las investigadoras toman el modelo de Kohn y Smith ${ }^{8}$, propuesta serial que supone que, a la representación semántica, siguen dos procesos. La investigación comprueba la hipótesis de Kohn y Smith sobre los dos niveles y realiza una discusión sobre este modelo. Concluye que la paciente observada presenta dificultades en la segunda etapa, no en la primera. Además, muestra que existe un predominio de la sílaba CV en la producción oral de la paciente; fenómeno condicionado, según las investigadoras, por la alta frecuencia de este tipo de sílabas en el idioma.

\section{Marco teórico}

\section{¿Un léxico; varios léxicos?}

Ellis $^{9}$ analiza la idea de que el lexicón presenta una organización específica y puede estar especializado, según se utilice

${ }^{7}$ Marina Laganaro y Anita Arrieta, «Representaciones fonológicas y fonémicas postlexicales en una afasia de conducción», Revista de Filología y Lingüística XXIV, 2 (1998): 207-219.

${ }^{8}$ Kohn y Smith (1984), citado en Laganaro y Arrieta.

9 Andrew Ellis, «La producción de palabras habladas desde la perspectiva de la neuropsicología cognitiva», Francisco Valle y otros (Eds.), Neuropsicología 
para producir el lenguaje o para percibirlo. Partiendo de evidencias obtenidas científicamente a partir de errores de habla de sujetos comunes, diversos estudios muestran que esta organización se basa, a lo sumo, en un eje semántico y uno fonológico ${ }^{10}$. Ellis ${ }^{11}$ plantea que podría suponerse la existencia de un léxico específico para la producción de palabras, organizado según las relaciones semánticas, puesto que la producción inicia en la etapa conceptual, en la que el hablante elabora un conjunto de proposiciones prelinguísticas relacionadas con el nivel del significado $^{12}$. Esto, al mismo tiempo, implicaría que también existe un léxico de percepción, organizado por la forma fonológica, puesto que son las señales acústicas y sus correspondencias fonológicas, los primeros estímulos que el oyente recibe en el momento del habla.

Por su parte, Allport ${ }^{13}$ pone en entredicho la existencia de dos lexicones fonológicos independientes. Postula la existencia de un único léxico basándose en: a. los errores de habla de los sujetos normales; b. los errores de habla de los sujetos afásicos; y c. un experimento llevado a cabo con tres pacientes afásicos. Allport retoma las ideas de Fay y Cutler $^{14}$, quienes suponen que, si existieran dos léxicos diferentes para producción y comprensión, no habría razones para que aparezcan confusiones fonológicas en la producción de

cognitiva del lenguaje. Lecturas de psicolingüística, Vol. 2 (Madrid: Alianza, 1990) 125-157.

${ }^{10}$ Mercedes Belinchón, Ángel Rivière y José Manuel Igoa, Psicología del lenguaje. Investigación y teoría, $3^{\mathrm{a}}$ ed. (Madrid: Trotta,1996); Jean Berko y Nan Bernstein (Eds.), Psicolingüística, $2^{\mathrm{a}}$ ed. (Madrid: McGraw Hill/Interamericana de España, 1999.

${ }^{11}$ Ellis, 141-142.

${ }^{12} \mathrm{La}$ mayoría de modelos propuestos para la producción del habla coinciden en postular una etapa de concepción prelingüística de ideas, que corresponde al momento en que el hablante piensa algo que quiere decir. También coinciden en proponer una fase de recepción fonológica en la percepción, que corresponde a la identificación del estímulo auditivo y la correspondencia que hace la mente con los fonemas apropiados (Berko; Belinchón, Rivière e Igoa).

${ }^{13}$ Allport, 212.

${ }^{14}$ Fay y Cutler (1977), citados en Allport, 213-4. 
palabras. Es decir, si se postula la existencia de un léxico de producción organizado por la relación semántica, no es lógico pensar que durante el proceso aparezcan errores de tipo fonológico, que serían solo probables durante la percepción. La conjunción de unos efectos semánticos y fonológicos mostraría la existencia de un solo lexicón.

En un segundo grupo de observaciones, Allport muestra las pruebas neuropsicológicas que ha hallado en torno a esta discusión; no se ha encontrado ninguna patología que presente un trastorno en que se haya dañado totalmente el léxico de producción y no el de comprensión o viceversa, lo cual es un indicativo fundamental para defender la existencia de un único conjunto léxico. Para Allport, es frecuente encontrar pacientes con una combinación tanto de dificultades receptivas como de expresivas, lo que supone un único lexicón.

Finalmente, analiza el caso de tres pacientes afásicos y demuestra que el deterioro de la forma fonológica de las palabras afecta tanto la comprensión como la producción. Concluye que existe un único lexicón, prueba más de economía cognitiva, es decir, de la optimización de recursos que realiza la mente humana para efectuar distintos procesos $^{15}$.

\section{Configuración del léxico mental}

Según Belinchón, Rivière e Igoa $^{16}$, cada una de las representaciones léxicas presentes en la mente del hablante, incluye los siguientes tipos de información:

1. una representación fonológica que especifica la estructura segmental de la palabra (secuencia de fonemas con rasgos distintivos), las sílabas que la forman y su patrón de acentuación;

${ }^{15}$ Allport, 214-225.

${ }^{16}$ Belinchón, Rivière e Igoa, 372. 
2. una representación ortográfica que implica una secuencia de letras descrita a partir de rasgos visuales; en este sentido se habla de alógrafos, es decir, de variantes estilísticas de una misma letra, identificada a partir de sus rasgos compartidos:

3. una representación morfológica que refleja la estructura derivativa de la entrada léxica y, en ciertos casos, su categoría gramatical;

4. una representación sintáctica que establece la categoría gramatical de la entrada y sus marcos de subcategorización;

5. una representación semántica consistente en un conjunto de rasgos primitivos semánticos o un enunciado-definición;

6. una lista de términos o conceptos asociados por el significado.

Esta propuesta supone que los hablantes tienen acceso a informaciones diversas sobre la palabra, conocimiento que se puede manifestar notoriamente en los errores espontáneos del habla y en la presencia reiterada, sistemática y característica, de confusiones léxicas por parte de los pacientes afásicos.

Por su parte, Fernández y Anula ${ }^{17}$ plantean que toda entrada léxica puede participar de una representación de los sonidos que componen la palabra (su forma fonética), una representación semántica (su significado) y una representación sintáctica (su categoría gramatical). Según Cuetos ${ }^{18}$, los sistemas que se plantean la existencia de cuatro listados léxicos distintos establecen que en cada uno se guardan las representaciones relativas a la función lingüística en la cual el módulo se ve involucrado, esto es, que el hablante guarda un listado de formas ortográficas para la escritura, un conjunto de formas visuales para la lectura, un conjunto de formas auditivas para la percepción/comprensión y un conjunto de formas fonológicas para la producción.

\section{Metodología}

${ }^{17}$ Marina Fernández Lagunilla y Alberto Anula Rebollo, Sintaxis y cognición. Introducción al conocimiento, el procesamiento y los déficits sintácticos. (Madrid: Síntesis, 1995).

${ }^{18}$ Cuetos, 1998. 
Para el estudio, se trabajó con el caso único de la paciente C.C. Antes de las pruebas, la paciente fue evaluada con la Batería Neuropsicológica de Barcelona y se descartaron problemas en el sistema conceptual. Las tareas que C.C. realizó para esta investigación se basaron en el estudio, el análisis y la reelaboración de pruebas según Goodglass y Wingfield; Ellis; Meyer, Schvaneveldt y Ruddy; Millar y Howard y Orchard-Lisle (1984) ${ }^{19}$.

\section{Obtención de los datos}

Se realizó una prueba de asociación semántica con cincuenta mujeres de edad entre 35 y 45 años, universitarias, considerando que C.C. es una mujer de 39 años, con estudios parauniversitarios. Para ejecutarla, se elaboró una lista de términos basada en el compendio léxico de Alameda y Cuetos $^{20}$ : «Frecuencia, longitud y vecindad ortográfica de las palabras de 3 a 16 letras del Diccionario de la Lengua Española». Este compendio ofrece un listado de todas las palabras del español registradas en el DRAE organizadas por número de grafemas y caracterizadas por su frecuencia de uso y otros datos estadísticos. La lista se elaboró tomando los primeros 15 sustantivos concretos e imaginables de cada listado. En total, se seleccionaron los primeros 170 sustantivos de frecuencia superior a 1, concretos, familiares e imaginables.

Una vez obtenidos los resultados de asociación semántica, se realizó un análisis estadístico de los datos. La lista inicial se redujo a partir de los siguientes criterios:

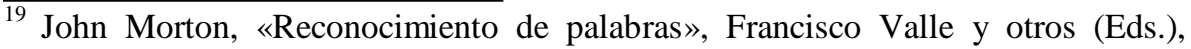
Comprensión y producción del lenguaje. Lecturas de Psicolingüística (Vol. 1) (Madrid: Alianza, 1990) 99-137; Merrill Garrett, «Percepción de palabras y oraciones», Valle y otros, 139-154.

${ }^{20}$ J. R. Alameda y Fernando Cuetos, «Frecuencia, longitud y vecindad ortográfica de las palabras de 3 a 16 letras del Diccionario de la Lengua Española», Revista Electrónica de Metodología Aplicada VIII, 2 (2003): 1-10. 
1. elección de los términos que presentaban una coincidencia superior a 20 en los lexemas asociados;

2. eliminación de las palabras de alta frecuencia e imaginabilidad que aparecían como asociación semántica de otras (agua, para río o mar);

3. eliminación de las palabras que tenían el mismo morfema raíz (niña-niño);

4. eliminación de extranjerismos o nombres comerciales como términos asociados (hamburguesa: McDonald's o Coca Cola);

5. eliminación de palabras ambiguas (coche);

6. sustitución de palabras del español peninsular por sus correspondencias costarricenses (computadora por ordenador, carro por coche-automóvil); y

7. eliminación de las palabras meta cuyos términos asociados presentaban el mismo morfema raíz (cafetería-café, salvavidas-salvar).

La lista resultante constó de 114 términos. Se indicó cada palabra meta con su correspondiente estímulo fonológico (sílaba inicial de la palabra) y estímulo semántico (palabras obtenidas en prueba de asociación semántica). Para cada palabra meta, se creó un estímulo visual que consistió en una fotografía real, de tamaño 14 x 19 $\mathrm{cm}$., presentada en computadora. Algunas imágenes fueron tomadas de Internet por la dificultad de adquisición que presentaba el referente (vg. murciélago, ejército, isla).

Antes de ser efectuada con C.C., la prueba se aplicó a un grupo control de diez sujetos sin patología, de edad y sexo similares a los de la paciente. Esto permitió descartar las fotografías que presentaban ambigüedades. Posteriormente, se realizaron las sesiones con la paciente. Se le presentó una única lámina por intento de denominación y se le dio tiempo ilimitado para contestar. Puesto que C.C. respondió a algunas palabras con facilidad, se le proporcionaron las claves fonológicas o semánticas solo en los casos en los que presentó dificultades. Se tomó como fin de intento de denominación, la palabra producida por la paciente o sus comentarios metalingüísticos en que 
expresamente indicó su imposibilidad para nombrar la imagen. En ese caso, se continuó con la imagen siguiente.

\section{Transliteración y transcripción de los datos}

La entrevista se grabó en casetes y luego se transliteró completamente, teniendo en cuenta los falsos comienzos, los titubeos y las pausas, los enunciados metalingüísticos y las intervenciones de la investigadora. En los casos en que se presentaron parafasias fonológicas, se procedió a la trascripción fonética.

\section{Análisis de los datos}

Se identificaron las siguientes clases de palabras:

1. palabras 1-0: palabras producidas correctamente, enunciados completos, fonológicamente correctos $\mathrm{y}$ producidos sin aproximaciones.

2. palabras 0-1: palabras producidas luego de intentos de enunciación (aproximaciones, silabeo, parafasias y circunloquios).

3. palabras 0-0: palabras no producidas o producidas parcialmente.

Para cada grupo de elementos léxicos, se determinó su organización por frecuencia, su organización por longitud, su organización por número de sílabas, su frecuencia promedio, su longitud promedio y su rango silábico (Casasa, en prensa). Además, se realizó un análisis cualitativo de la información, del cual se obtuvieron los siguientes resultados.

\section{Análisis de los datos}

Etapas del proceso de producción: acceso independiente a los módulos semántico y fonológico

A partir del análisis de los datos obtenidos en las entrevistas con la paciente, se infiere que la información semántica y la fonológica se activan en dos etapas diferentes y podría considerarse que pertenecen a dos módulos distintos. Como se observa en el 
ejemplo (1), la paciente evidencia su conocimiento del objeto y de algunas características semánticas asociadas con él ${ }^{21}$.

(1)

pared

P: Eh... pue... eh... No, eso se llama... ay, este... ay, ¿cómo es que se llama?... Mi papá me lo hizo.

E: ¿iSí?

P: Sí, el me... Él fue el que me hizo la casa.

E: Ajá.

P: Pero ya no me acuerdo cómo se llama eso.

E: Sí. La pista es... ajá. El sinónimo, un sinónimo es muro.

P: Muro... ah, muro, muro, muro.

E: Ajá, ¿y otra forma de decirle? ¿Como esta?

P: ¿Aquí?

E: ¿Esto?

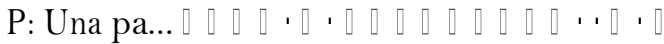

\section{columna}

P: Eso es una... este... es como un edificio... pero... pero como... como en Heredia que hay un... coso allá, yendo por a... iglesia que se llama... digo yo porque lo veo parecido... un de los de antes, de años, ¿verdad? Eh... No, no sé cómo es, pero yo sé lo que es, pero no sé el nombre.

Según esto, en el proceso de producción de lenguaje ocurriría un primer estadio de acceso a la información semántica de la palabra. Esta primera etapa de producción linguiística correspondería a la activación del concepto en el sistema semántico. Parece que existe un déficit en el proceso que llevaría esta información semántica a la localización de una determinada forma fonológica.

${ }^{21}$ La notación de los ejemplos sigue los siguientes criterios: en primer lugar, existe un número que servirá como referencia del ejemplo; seguidamente, aparece la palabra meta señalada con letra negrita; inmediatamente, se muestra el diálogo correspondiente, donde $\mathrm{E}$ es entrevistadora y $\mathrm{P}$, paciente. Se mantiene la trascripción fonética para las parafasias fonológicas y el uso del punto para indicar las pausas. 
La información de parte de la paciente indica que, durante el proceso de producción, la etapa semántica es anterior a la localización de la forma fonológica de la palabra. Además, la hablante establece ciertas relaciones con otras palabras de su conocimiento y también con ciertos rasgos del referente, antes de llegar a la palabra meta.

Además, las muestras de habla tomadas de la entrevista sugieren la existencia de dos planos distintos de relaciones. Los datos indican que la paciente reconoce el significado de la palabra a partir de otras relaciones, las cuales se podrían considerar nodos relacionados o rasgos constituyentes de la forma léxica; ver (2).

\section{sacerdote}

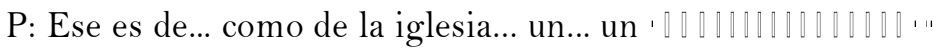

\section{radio}

P: En un carro... Eleca... el... el... En un carro un radio... Bueno, un radio. No sé cómo es que se llama. ¿Verdad que es de carro?

E: Radio está bien.

\section{ventana}

\section{P:0000001000 No.00001000000 Ayllo0000000100

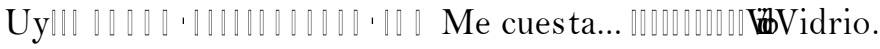 \\ 0000101000.00000 Ventana, ventana, ventana.}

mano

P: Una... cinco ojos, eh... perdón. Cinco dedos... eh... una mano.

Para «mano» se presenta una especie de definición que parte de la enunciación de ciertos componentes indispensables del objeto. Así, podemos encontrar dos tipos de relaciones semánticas en ambos ejemplos, las que $\operatorname{Leech}^{22}$ denomina significado conceptual y significado asociativo. En el primer caso, hablamos de aquel significado que se establece a partir de rasgos contrastantes marcados de manera positiva o negativa; en el segundo, de aquellos términos asociados en la contigüidad de las percepciones de los hablantes; es

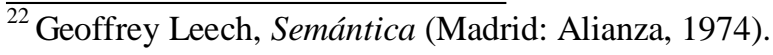


decir, que las unidades léxicas establecen, en una misma etapa de procesamiento, un conjunto de relaciones con otras unidades léxicas (relaciones de tipo asociativo) y la activación de un conjunto de rasgos elementales (relaciones de tipo conceptual), que bien podrían ser una categoría específica y delimitada (tal como lo plantea la teoría de rasgos), o bien, podrían ser un conjunto difuso, sin límites precisos, pero que sí contendría ciertas características básicas o típicas del referente (tal como lo propone la teoría de los prototipos).

Estas relaciones de tipo asociativo explican la aparición de otras unidades léxicas relacionadas en el esquema de mundo del hablante. La hablante utiliza circunloquios para referirse a realidades que no logra nombrar. Retomando las ideas de Goodglass y Wingfield $^{23}$, podrían ser interpretarse como autosugerencia, o bien, siguiendo a Sánchez y Martín ${ }^{24}$, como un error en el sistema de procesamiento, en el que la información semántica debería encontrar una representación fonológica pero no lo logra. El circunloquio establece relaciones de tipo paradigmático con la palabra meta. En (2) se relacionan, por ejemplo, «sacerdote» con «iglesia», «radio»con «carro» o «ventana» con «vidrio», mientras que (3) muestra cómo los circunloquios se establecen a través de relaciones paradigmáticas de unidades léxicas asociadas.

\section{(3)}

\section{jabón}

P: Irex. Polvo de la... de lavar... Sí, polvo de... la... lavar.

E: ¿Cómo se llama?

P: ¿Cómo se llama? ¿I ere? ¿Irés? ¿Irex o...? ¿Polvo? Sí, polvo de... ¿ipolvo?

E: Palabra lavar... detergente.

$P:$

E: Y también se llama ja...

\footnotetext{
${ }^{23}$ Harold Goodglass y Arthur Wingfield (Eds.), Anomia. Neuroanatomical and Cognitive Correlates (San Diego: Academic Press, 1997).

${ }^{24}$ María Luisa Sánchez Bernardos y Pilar Martín, «El enfoque psicolingüístico en el estudio de la patología del lenguaje», L. Manning (Ed.), Introducción a la Neuropsicología clásica y cognitiva del lenguaje (Madrid: Trotta, 1992) 141-186.
} 


\section{P:}

Se pueden postular dos tipos de relaciones semánticas. Las relaciones asociativas confirman las teorías conexionistas del léxico ${ }^{25}$ que plantean que las unidades lingüísticas se establecen mediante conexiones entre distintos nodos de información, correspondientes a diferentes tipos de representaciones. Eso explica que en lugar de que la paciente produzca la palabra meta, se reproduzca una palabra que mantenga con ella una relación semántica asociativa o el circunloquio. El módulo semántico del sistema cognitivo se podría pensar articulado en forma de redes o conexiones. A la vez, antes de recuperar las unidades léxicas correspondientes, ya se han activado los rasgos componenciales relacionados con el referente. En el proceso, estos mismos rasgos enviarían información a las otras unidades léxicas relacionadas, de manera que aunque no se haya activado la palabra meta, sí se han activado las otras unidades léxicas completamente. Algunos autores afirman la participación del efecto conocido como «priming» semántico o facilitación. Este fenómeno implica que en pruebas experimentales, el significado de una palabra es reconocido más pronto cuando antes se ha propuesto al paciente una palabra semánticamente relacionada ${ }^{26}$. En este sentido, la palabra «pantalón» del ejemplo (4) podría haber resultado facilitada por la denominación previa de «zapato». La perspectiva conexionista se confirma con la presencia de parafasias.

\section{pantalón}

P: Es za... zapato. Eh... un... pantalón...

\section{El flujo de información}

\footnotetext{
${ }^{25}$ Lauretta Reeves, Katty Hirsh-Pasek y Roberta Golinkof, en Berko y Bernstein, 193-4.

${ }^{26}$ Cuetos, 28; Belinchón, 398-9.
} 
El proceso lingüístico se ha descrito de forma serial o interactiva $^{27}$. En los modelos seriales, los subprocesadores se disponen en una estructura jerárquica y funcionan de forma secuencial. Cada uno recibe información de los subprocesadores directamente conectados a él. El flujo de información en el sistema discurre unidireccionalmente. Por su parte, el modelo interactivo plantea la interacción entre los diversos subsistemas de procesamiento; defiende el carácter paralelo y simultáneo de los procesos de actuación lingüística. Una propuesta intermedia es el modelo en cascada, que supone que el flujo de información no espera a terminar un proceso para iniciar el siguiente. Estos modelos se analizan a continuación, a la luz de la entrevista con C.C. (5).

(5)

\section{escalera}

P: Eso es un...eh...este...Que esta me cuesta tan... no sé cómo...

E: Ahí sería subir... es la palabra relacionada...

P: Subir... este... los... los...

E: Empieza con es...

P: Es...es... no.

hotel

P: Un

E: Hospedaje... se relaciona con hospedaje.

P: Hospital, eh... sí, hotel, bueno, hotel, sí, hotel o... sí.

Cuando se ofrece a la paciente un estímulo de tipo semántico, su primera reacción característica es repetir el estímulo. Este estímulo parece no ofrecer mayores claves lingüísticas para dar con la forma de la palabra. El estímulo «hospedaje», cuando la palabra meta es «hotel», motiva la respuesta «hospital», cuya estructura fonológica inicial es semejante a la del estímulo, si bien se podrían encontrar relaciones semánticas entre «hospedaje» $\mathrm{y}$ «hospital». Ante estímulos semánticos, la paciente utiliza como índice de búsqueda, en primer lugar, la estructura fonológica. Los datos indican que la información

$\overline{{ }^{27} \text { Belinchón, Rivière e Igoa; Cuetos. }}$ 
semántica permanece en el nivel de lo semántico y que esta información es poco efectiva para hallar la forma de la palabra que se busca. Se trataría de dos tipos de representaciones distintas (representaciones fonológicas y semánticas), pertenecientes a dos estadios también distintos de procesamiento. Los datos parecen reflejar un flujo de información serial (o, eventualmente, en cascada), pero de ningún modo interactivo.

\section{Estímulos semánticos y fonológicos durante el acceso léxico}

Se ha observado que los estímulos dados a la paciente, tanto fonológicos como semánticos, condicionan respuestas de tipo fonológico. En este sentido, la etapa de formulación formal de la palabra meta se realiza a partir de información fonológica. En esencia, las claves fonológicas son más efectivas en el hallazgo de la palabra meta que las claves semánticas. Por ello, se trata de dos distintos estadios de procesamiento en los que las representaciones y su organización son de naturaleza distinta. Si bien la paciente conserva y reconoce las características semánticas de los lexemas, el que no llegue a la forma fonológica que busca se debe a que esta se encuentra en un estadio posterior de información y presenta características fonológicas de organización.

\section{Elementos suprasegmentales}

Distintos elementos suprasegmentales acompañan los diversos intentos de producción de la paciente: las aproximaciones presentan alargamiento de segmentos fonológicos, silabeo, nasalización y cambio acentual. Obsérvese el ejemplo (6):

(6)

\section{mantequilla}

P: Numar, numar, sí, numar, no, o...

E: El nombre general.

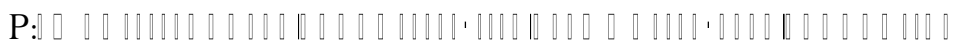


P: Uy, me cuesta la... la que me falta al final...

calzoncillo

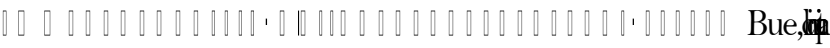

P: Ese es un... este... ay... lo de los hombres, este...eh....cal... cal.zon.ci.llo.

desodorante

P: Eso... es de... sí... este... se llama...

sangre

$\mathrm{P}: \llbracket$

esqueleto

P:

En la reconstrucción de la forma fonológica, la paciente introduce variaciones de propiedades suprasegmentales y segmentales. Esto conduce a la hipótesis de que estas variaciones están asociadas con estadios de reconstrucción de la forma fonológica y que permiten a la paciente elaborar de manera sucesiva la forma productiva que corresponde con su intención.

Por otra parte, el silabeo permite la configuración de la palabra, como en el caso de «mantequilla» o «esqueleto». Como apuntan Belinchón, Rivière e $\operatorname{Igoa}^{28}$ : «la principal virtualidad de la sílaba radica en su condición de organizador de las unidades segmentales o fonémicas en unidades rítmicas y melódicas». Estos datos suponen una confirmación de la realidad psicológica de la sílaba como unidad abstracta de organización fonémica. Además del silabeo, la duda sobre la forma de la palabra se manifiesta en alargamientos consonánticos y vocálicos, como se observa en «desodorante»o en «sangre».

Los procesos realizados por C.C. pueden considerarse intentos en los que el sujeto confronta su propia producción con la «huella» de la forma fonológica que conserva en su almacén léxico. Cuando se logra producir la palabra meta, desaparecen los intentos marcados por

${ }^{28}$ Belinchón, Rivière e Igjjoa, 599. 
estos rasgos. El proceso está marcado por la elaboración de aproximaciones, caracterizadas por parafasias fonológicas que presentan variaciones acentuales, alargamiento de segmentos, silabeo y nasalización. Se puede suponer, entonces, que la paciente busca conjugar en un mismo plano de construcción del elemento léxico los patrones silábicos, prosódicos y segmentales apropiados. Véase al respecto el ejemplo (7).

\section{murciélago}

P: Ay, ese bicho. 0000000001100000 Yo sé que le falta una palabraoalgo así. 0 0001000101010 Uy, me cuesta la palabra.

\section{policía}

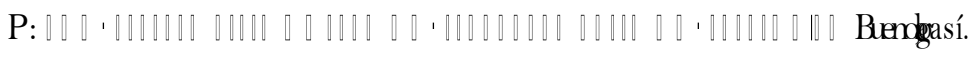

\section{Organización de las representaciones fonológicas}

La entrevista muestra que las diferentes ayudas fonológicas que se ofrecieron a la paciente generan distintas formas de producción. Analicemos (8), donde tenemos un conjunto de claves fonológicas que consisten en la sílaba inicial de la palabra.

\section{maquillaje}

P: Este, eso es en los di... en la... cara, en eh... en el... esto... ¿cómo se llama?... en la boca... se llama... labio... labios... por los ojos... aquí y por el resto de toda la cara.

E: Empieza con ma.

P: Ma. ¿Esto?

E: O en Costa Rica también le decimos pint...

P: ¿Pint? ¿Pintura?

E: Ajá. Así le decimos aquí. Y luego, hay otra palabra que es la que como en... uno va a la tienda y busca ma... o dan clases de ma...

P: Ma... no.

isla

P: Montaña.

E: Cuando...a ver esa. 
P: Edificios, no.

E: Is...

P: ¿Cómo?

E: Is...

P: ¿Is?

E: Cuando hay tierra y alrededor agua, ¿es una is...?

P: Is... isla, isla.

\section{jabón}

P: Irex. Polvo de la... de lavar... Sí, polvo de... la... lavar.

E: ¿Cómo se llama?

P: ¿Cómo se llama? ¿I ere? ¿Irés? ¿Irex o...? ¿Polvo? Sí, polvo de... ¿polvo?

E: Palabra lavar... detergente.

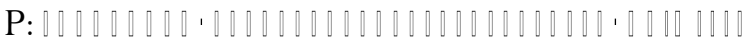

E: Y también se llama ja...

$\mathrm{P:}$

En los ejemplos anteriores, la clave silábica obtiene buenos resultados en el caso de «isla». Lo mismo ocurre cuando la palabra meta es «jabón». Podríamos figurarnos que la longitud de la palabra interviene en la facilidad de recuperación, además de considerar que, en el caso de las palabras bisílabas, cuando se le ofrece a la paciente la sílaba inicial del elemento léxico, se le está guiando en la mitad del proceso de recuperación. No ocurre lo mismo con la palabra «maquillaje», cuya complejidad es mayor que la de los términos anteriores. En efecto, la paciente logra recuperar con acierto las palabras de menor longitud.

Consideremos ahora (9), vinculado con el posible tipo de relaciones que se establecen a partir del segmento fonológico dado.

\section{pasillo}

P: Eso es una... una parte como decir... eh... ¿cómo se llama?

E: Empieza con pa.

P: ${ }_{\complement} \mathrm{Pa}$ ? Parada... no. Pa... pa... una pa... una pa, ¿iverdad?... Para... no... no... pa... nos... no... 
En este ejemplo, el estímulo activa una palabra que presenta una estructura inicial similar. De esta manera, el resultado del estímulo fonológico se homologa con los efectos de los estímulos semánticos: tal como habíamos visto, prima la recuperación fonológica tanto cuando se presentan estímulos semánticos, como cuando se dan estímulos fonológicos. La recuperación de "parada», en este caso, que desde el punto de vista conceptual no presenta elementos comunes con «pasillo», puede ser un indicio de la recuperación de «vecinos fonológicos» por parte de la paciente. Las teorías conexionistas, que proponen la activación de varios elementos linguísticos candidatos durante el proceso del habla, indican que el léxico fonológico se organiza por la similitud fonológica ${ }^{29}$. Esto podría explicar la aparición de la forma $\square \cdot \gamma \square \square \beta \square$ en sustitución de «fuego», que presenta el núcleo vocálico y un patrón de acentuación cercanos, o la aproximación la paciente al intentar producir la pllameta «estrella» (10).

(10)

fuego

P: Eso es qué $\square \gamma \square \square \beta \square$, no... Fuego, no. ¿¿Fuego, verdad, o no? Sí, fuego. estrella

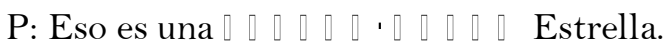

Las propuestas conexionistas demuestran que existen nodos de información interconectados y que estos se activan de manera simultánea ante un estímulo. En el caso de «parada/pasillo», lo que ocurriría es que el estímulo ha activado una forma fonológica competitiva, que no es inhibida por la paciente durante el proceso de producción. Ahora bien, el código de acceso que permitiría elegir el candidato correcto se ha denominado punto de unicidad.

\section{El punto de unicidad como código de acceso léxico}

$\overline{{ }^{29} \text { Reeves, Hirsh-Pasek y Golinkoff, }} 195$. 
Muchos investigadores coinciden en que el acceso al léxico mental está guiado por la porción inicial de la palabra, así que la tarea principal de la investigación ha sido delimitar cuáles elementos de esa porción inicial intervienen en el proceso $^{30}$. El código del acceso léxico, conocido como punto de unicidad, se ha homologado en distintas propuestas con el fonema inicial de la palabra, su sílaba inicial fonológica, su sílaba inicial ortográfica, su primera sílaba acentuada o su terminación. Los modelos suponen que el punto de unicidad se obtiene por un proceso de segmentación de la entrada sensorial en unidades lingüísticas discretas. También se ha demostrado que el código empleado en el acceso léxico no sigue pautas universales, sino que depende de las características idiosincráticas del sistema fonológico de la lengua materna ${ }^{31}$.

La preponderancia del segmento inicial de la palabra se observa en distintas emisiones de la paciente, como muestra (11), en que ella misma reconoce que son los segmentos fonológicos finales los que le presentan mayor dificultad.

\section{(11)}

\section{insecticida}

P: Baygón.

E: ¿Y el otro nombre?

P: Este... eh... como en

\section{calendario}

00800000000000000000000000000000000 Ah, la maparte...

P: El mes... la... ¿cómo se llama? Sí, por para meses, para cada mes, un mes, digamos, que se ponen los $\square \square \square \square \square \square \square$, los números.

E Un ca...

P número...

E:

\footnotetext{
${ }^{30}$ Belinchón, Rivière e Igoa; Berko y Bernstein.

${ }^{31}$ Belinchón, Rivière e Igoa, 380.
} 


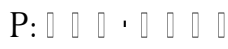

E:000.000

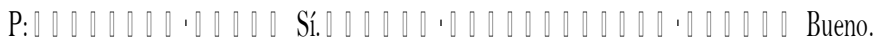

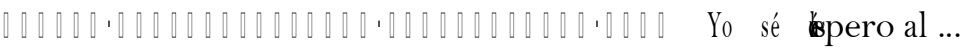

aunque estoy terminando al final...

En (11), en un primer momento la entrevistadora ofrece, como estímulo fonológico, la sílaba inicial de la palabra meta, pero luego, ante las dificultades de la entrevistada, va incorporando fonemas sucesivos. Conforme la estructura fonológica se desarrolla, crecen las posibilidades de acceso a la palabra. La sílaba inicial de la palabra no corresponde en todos los casos con el reconocimiento. De diez lexemas en los cuales se le dio a la paciente una clave fonológica, únicamente tres fueron localizados a partir de la sílaba inicial. Uno de ellos era un elemento que había sido mencionado por la paciente con anterioridad, lo cual nos hace pensar que ya presentaba determinado nivel de activación. Como hemos mencionado, los otros dos lexemas presentan una estructura de dos sílabas, longitud que favorece el hallazgo del término. Así, los datos confirman la existencia de un punto de unicidad (que no en todos los casos corresponde a la sílaba inicial), a partir del cual surge la localización de la palabra.

(12)

escalera

E: Ahí sería subir... es la palabra relacionada...

P: Subir... este... los... los...

E: Empieza con es...

P: Es...es...no.

E: Esca...

P:0000000000000000000100000000000

E: Escal...

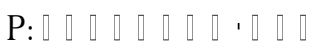

E: Escale...

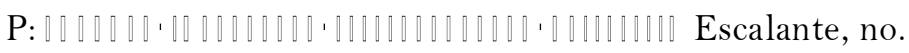


E: Escale...

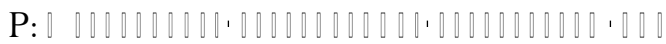

\section{columna}

E: Esta es de arquitectura. Es lo que sostiene, ¿verdad?. Sirve como para sostener. También tenemos aquí atrás. La llamamos igual.

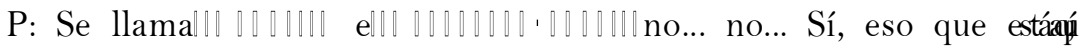
desde aquí hacia, hacia... atrás.

E: Empieza con col...

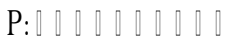

E: Colu...

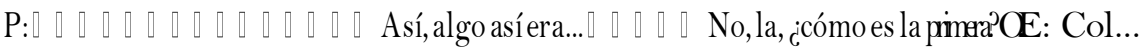

P:०००००

E: Colu...

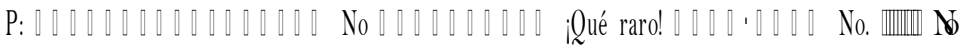

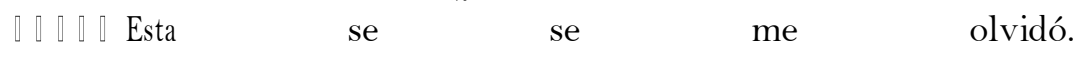

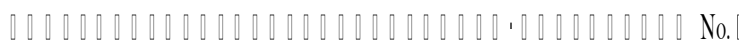

E: Ajá, correcto.

En (12), se observa que el punto de unicidad corresponde en dos casos con el segmento inicial de la palabra hasta la sílaba acentuada («colum»y «escale»). Por otra parte, también surge como punto de unicidad un segmento correspondiente a dos sílabas iniciales de palabra no acentuadas (ver (11), «calendario»). Dado que los datos no presentan un patrón sistemático y que son muy pocos, no se puede establecer una conclusión definitiva sobre este aspecto. Sin embargo, parece que este punto de unicidad le permite a la paciente descartar aquellos candidatos que compiten con la palabra meta.

\section{Patrón silábico de la palabra meta: explicaciones desde la adquisición del lenguaje}

En la entrevista se ha observado que la paciente conserva el reconocimiento de la estructura prosódica y silábica. Asimismo, en 
esta etapa parece existir una simplificación de las sílabas manifestada en la preferencia por estructuras $\mathrm{CV}^{32}$.

Las teorías de adquisición del lenguaje establecen que la sílaba $\mathrm{CV}$ es adquirida en primer lugar, seguida por la sílaba formada únicamente por el núcleo V. Según Serra y otros, la simplificación de la estructura silábica durante la infancia tiende a realizarse siguiendo los procesos de omisión de la coda y reducción de los grupos consonánticos de ataque ${ }^{33}$. Asimismo, el ataque y el núcleo de las sílabas son percibidos e identificados por los niños mejor que la coda. En este sentido, cabría preguntarse si la tendencia a formular este tipo de estructuras no corresponde a una reelaboración de las etapas de adquisición del lenguaje, de manera que el habla adulta afásica de la paciente acude a los mismos mecanismos utilizados en las primeras etapas del desarrollo linguístico. Ahora bien, habría que explicar por qué no suceden etapas iniciales como el gagueo, el juego vocálico o el balbuceo.

El balbuceo, que ocurre durante los primeros seis meses de vida, se caracteriza también por la presencia de sílabas CV. Incluso los procesos de mantenimiento, afinación y pérdida de la estructura silábica durante el balbuceo son parecidos en todas las lenguas y muestran un carácter universal ${ }^{34}$. Suponemos que el sistema lingüístico de la paciente tiene consolidadas estas etapas del sistema fonológico y no presenta pérdida en ellas; es decir, que en la producción de la paciente hay un énfasis en la reestructuración de la sílaba que permite, posteriormente, acceder a la totalidad de la forma fonológica, pero que no va a etapas iniciales del desarrollo fonológico.

\section{Estructuras vocálicas en la producción fonológica: tendencias a la neutralización y recuperación de estadios de evolución del sistema fonológico}

\footnotetext{
${ }^{32}$ Cfr. con Laganaro y Arrieta.

${ }^{33}$ Miquel Serra y otros, La adquisición del lenguaje (Barcelona: Ariel, 2000) 209.

${ }^{34}$ Serra, 191-2.
} 
Además de los fenómenos descritos, hemos observado la tendencia a una búsqueda de la armonía vocálica ((12) «columna» y (13)) y a la utilización del fono $[\square]$ que podría corresponder a una manifestación afásica adulta de las etapas de construcción de los segmentos vocálicos.

chocolate

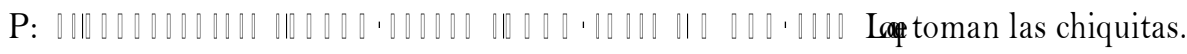
desodorante

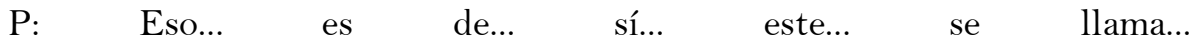

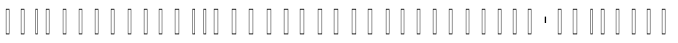

El fono [ $\square]$, caracterizado por ser un segmento central, se manifiesta como una realización neutra de los segmentos vocálicos aún no identificados por la paciente. Esta característica parece dar cuenta de un estadio de formación silábica en que los segmentos aún no se han precisado y en que la paciente tiende a la recuperación de formas neutras.

Además, la armonía vocálica vista como una etapa de evolución del sistema fonológico humano, tal como propone Tomcsányi ${ }^{35}$, se presenta en la producción lingüística de la paciente como una forma de acercamiento a la palabra meta. Las teorías que plantean que la recuperación fonológica de la sílaba inicia con el núcleo como elemento estructurador y continúa con la recuperación del ataque $\mathrm{y}$, posteriormente, de la $\operatorname{coda}^{36}$, podrían explicar la aparición de parafasias vocálicas y la indeterminación, en algunos casos, de las formas consonánticas propias, manifestadas en anticipaciones y perseveraciones. En este sentido, ante la dificultad de

\footnotetext{
35 Judit Tomcsányi, «Armonía vocálica y construcción simbolizadora del lenguaje». Letras XXV-XXVI (1980): 237-254.

${ }^{36}$ Belinchón, Rivière e Igoa.
} 
recuperar, en primer término, el núcleo de la sílaba, la paciente recurriría a elementos neutrales o a la regulación de los rasgos vocálicos presentes, permitiendo, entonces, localizar los segmentos posteriores. El ataque (la parte de la sílaba que se formaría) se manifestaría con la recuperación de otros segmentos que también son parte de la forma fonológica, lo que se traduce en anticipaciones, perseveraciones, asimilaciones y disimilaciones (las aproximaciones de la palabra meta «mantequilla» presentan estos fenómenos, lo que da cuenta de que la paciente ha logrado recuperar algunos de los segmentos fonológicos o sus rasgos, si bien, todavía no los ha ubicado en la posición correcta). Finalmente, la coda se podría diluir o no identificar, lo que explicaría, también, la tendencia a las sílabas CV.

Todo ello tiene implicaciones de carácter teórico. Por una parte, sería una muestra evidente del uso por parte de la hablante de información fonológica correspondiente a etapas anteriores de su desarrollo lingüístico (vgr. la tendencia a la sílaba CV). Por otra, constituiría una evidencia de que la paciente también recurre a etapas anteriores en la formación del sistema fonológico, en que la vocal aparece como un segmento aún no diferenciado y no arbitrario (vgr. la búsqueda de la armonía vocálica). Finalmente, establece que el proceso serial de formación de sílaba plantea a la paciente la necesidad de formular un núcleo neutral, a saber, el segmento $[\square]$ que suple su imposibilidad de dar con el segmento adecuado al contexto.

\section{Conclusiones}

Se ha confirmado la existencia de distintas etapas en la producción del léxico; además, se ha observado el efecto que tienen los estímulos semánticos y fonológicos en la producción de palabras de la paciente. La paciente muestra una etapa de preparación y activación de información semántica, en la que no tiene acceso a la información fonológica. Esta etapa es previa a la activación de la forma de la palabra. En ella, las claves semánticas le proporcionan a la paciente indicios del significado y la ubicación del referente en el 
sistema conceptual, como muestra el hecho de que ella afirma reconocer el objeto y establece relaciones de significado conceptuales y asociativas a partir de ese reconocimiento. En esta etapa, la información semántica suministrada por la investigadora rige procesos de activación de significados, o bien, condiciona respuestas fonológicas en función de la estructura segmental del estímulo. Esto confirma la modularidad y la autonomía de los sistemas semántico y fonológico, tal como la propone el modelo de Morton. La etapa posterior, la fonológica, no se beneficia con los estímulos de orden semántico.

Estas evidencias hacen pensar que no existen dos corpus léxicos independientes e inclinan a desechar la idea de que los procesos de acceso léxico están guiados por la relación semántica. Según nuestro análisis, el conocimiento semántico es relevante durante los procesos de formulación semántica. Los datos indican que el proceso es serial y que el acceso a la información semántica rige el procesamiento de información relativa al significado, la cual, una vez obtenida, encuentra correspondencias fonológicas de salida con representaciones almacenadas en la mente del hablante en función de su forma. Se evidencia un flujo único de información que cambia de naturaleza en sus distintas etapas de procesamiento.

Sobre este aspecto, queda pendiente un análisis más minucioso sobre el concepto de palabra (o unidad léxica) que se ha mantenido en los tratados de psicolinguística; probablemente esta perspectiva ha obligado a concebir la palabra, no como una unidad estructurada y fijada en la mente del hablante, sino como la convergencia, en determinado punto del proceso cognitivo, de un cúmulo de representaciones dispersas. En este sentido, la producción fonética de «palabras» solo es una etapa más de transformación de ciertas unidades representacionales.

En relación con el acceso léxico, se observan tres etapas distintas de producción: conceptual, semántica y de recuperación fonológica. Durante la semántica se establecen relaciones conceptuales y asociativas a partir del estímulo visual, que permiten a la paciente 
reunir un conjunto de representaciones relacionadas con el significado. Estas asociaciones tienen carácter reticular y parecen responder a los modelos conexionistas de organización del significado aunque los datos no permiten asegurarlo totalmente. La ausencia de una correspondencia fonológica para este conjunto de representaciones semánticas, provocada por una alteración en el flujo de la información, conduce a la aparición del circunloquio, el cual adquiere una relación paradigmática con la palabra meta.

En relación con la etapa fonológica, se observan los mismos resultados obtenidos en estudios previos ${ }^{37}$ sobre la existencia de dos etapas de estructuración fonológica: por una parte, un estadio en que se ha elaborado la estructura silábica y prosódica de la palabra meta pero aún no se han adjudicado los segmentos correspondientes; por otra, en la que se asignan estos segmentos. Se ha observado en esa primera etapa de proyección estructural de la palabra, una tendencia a la producción de sílabas de tipo $\mathrm{CV}$, lo cual puede relacionarse con una posible recuperación de etapas iniciales de adquisición del lenguaje, como recurso de reconstrucción de las formas léxicas, en que son preponderantes las sílabas $\mathrm{CV}$.

También se corrobora la relevancia de la parte inicial de la palabra, que por lo general es conservada por la paciente y es significativa en los procesos de búsqueda que ella realiza. Estos segmentos iniciales se relacionan con el criterio de punto de unicidad (que permitiría el acceso léxico), pero los datos no permiten establecer una definición concreta sobre su naturaleza. Se puede descartar que la sílaba inicial de la palabra sea el punto de unicidad en todos los casos, solo es efectiva cuando se trata de palabras bisílabas. Por esto, se sugiere la relación que debe existir entre punto de unicidad y longitud de la palabra.

Ahora bien, la estructuración fonológica de la palabra meta presenta un conjunto de rasgos suprasegmentales asociados con los momentos de búsqueda realizados por la paciente y por su indecisión

${ }^{37}$ Laganaro y Arrieta. 
sobre sus mismos enunciados. Se ha afirmado que no se encuentran condicionamientos fonológicos en las parafasias producidas por la paciente y que, más bien, estos rasgos se presentan en momentos de duda, caracterizados por las pausas y las aproximaciones. Los rasgos observados son el silabeo, la nasalización de segmentos vocálicos, el aumento en la cantidad consonántica y silábica y el cambio acentual. Aquí se observa una vez más la preponderancia de la sílaba como elemento estructurador de la palabra y su realidad fonológica.

Dos procesos parecen mediar la producción de las palabras por parte de la paciente. Se ha identificado, por una parte, la tendencia a la utilización del segmento neutral [ $\square$ ], que permitiría configurar la estructura silábica a partir de la ubicación de un núcleo provisional y la recuperación posterior del ataque y la coda. Así se explicaría la aparición de este segmento vocálico durante las aproximaciones de la palabra (se trataría de un elemento configurador, sustituto momentáneo de la unidad vocálica buscada). La recuperación de los segmentos consonánticos, antes de la definición de su lugar en la estructura fonológica de la palabra meta, permite explicar la aparición de anticipaciones, intercambios, perseveraciones, asimilaciones y disimilaciones. La imposibilidad de recuperación de la coda es una explicación más en relación con la preponderancia de la sílaba CV. El segundo proceso se relaciona con la tendencia a la armonía vocálica. Si se concibe la armonía vocálica como una etapa de evolución de los sistemas fonológicos, en que se recurría a segmentos no diferenciados, tal como se planteaba antes, se puede suponer que la manifestación productiva de la palabra da cuenta de estadios anteriores de evolución fonológica, lo que constituye una huella de las transformaciones diacrónicas del sistema de procesamiento lingüístico universal. En este sentido, la recuperación léxica realizada por la paciente afásica reuniría, en su construcción paulatina y dificultosa, principios evolutivos y adquisitivos de construcción del lenguaje.

A lo largo del presente estudio se han trazado distintas líneas de investigación. Para el desarrollo de los estudios de psicolingüística en el país, es urgente la elaboración de índices de frecuencia, 
familiaridad, edad de adquisición y concreción de palabras del español de Costa Rica. En relación con el acceso léxico, sugerimos nuevos estudios que identifiquen con precisión el punto de unicidad que desencadena el acceso, los tipos de flujo de información que se dan en él y la naturaleza de las relaciones de sus representaciones. 